\title{
Dynamic Power Tariff for Congestion Management in Distribution Networks
}

\author{
Huang, Shaojun; Wu, Qiuwei; Shahidehpour, Mohammad ; Liu, Zhaoxi
}

Published in:

IEEE Transactions on Smart Grid

Link to article, DOI:

10.1109/TSG.2018.2790638

Publication date:

2018

Document Version

Publisher's PDF, also known as Version of record

Link back to DTU Orbit

Citation (APA):

Huang, S., Wu, Q., Shahidehpour, M., \& Liu, Z. (2018). Dynamic Power Tariff for Congestion Management in Distribution Networks. IEEE Transactions on Smart Grid, [1-10]. https://doi.org/10.1109/TSG.2018.2790638

\section{General rights}

Copyright and moral rights for the publications made accessible in the public portal are retained by the authors and/or other copyright owners and it is a condition of accessing publications that users recognise and abide by the legal requirements associated with these rights.

- Users may download and print one copy of any publication from the public portal for the purpose of private study or research.

- You may not further distribute the material or use it for any profit-making activity or commercial gain

- You may freely distribute the URL identifying the publication in the public portal 


\title{
Dynamic Power Tariff for Congestion Manage- ment in Distribution Networks
}

\author{
Shaojun Huang, Member, IEEE, Qiuwei Wu, Senior Member, IEEE, Mohammad Shahidehpour, Fel- \\ low, IEEE, Zhaoxin Liu, Member, IEEE
}

\begin{abstract}
This paper proposes dynamic power tariff (DPT), a new concept for congestion management in distribution networks with high penetration of electric vehicles (EVs), and heat pumps (HPs). The DPT concept is proposed to overcome a drawback of the dynamic tariff (DT) method, i.e., DPT can replace the price sensitivity parameter in the DT method, which is relatively unrealistic in practice. Based on the control theory, a control model with two control loops, i.e., the power flow control and voltage control, is established to analyze the congestion management process by the DPT method. Furthermore, an iterative method based on distributed optimization is proposed to determine the DPT rates, which enables active participation of aggregators in the congestion management. The case studies demonstrate the efficacy of the DPT method for congestion management in distribution networks, and show its ability to save congestion management cost compared to the DT methods.
\end{abstract}

Index Terms-- Congestion management, distribution system operator (DSO), distributed optimization, dynamic power tariff (DPT), electric vehicle (EV), heat pump (HP).

\section{NOMENCLATURE}

\section{Parameters}

$A_{i, t, t_{-}}$

coefficient matrix, describe the relations between the power consumption and temperature change of the household

$D \quad$ power transfer distribution factor (PTDF)

$\tilde{E}_{i, t}, E_{i} \quad$ customer to load bus mapping matrix

$F_{t} \quad$ line loading limit of active power

$K_{i, t}^{a, \min } \quad$ lower temperature limit

$K_{i, t}^{a, \max } \quad$ upper temperature limit

$N_{B} \quad$ set of aggregators

$N_{T} \quad$ set of planning periods

$N_{L} \quad$ set of lines

$N_{d} \quad$ set of demand bus

V voltage lower limit

S. Huang, Q. Wu and Z. Liu are with the Center for Electric Power and Energy (CEE), Department of Electrical Engineering, Technical University of Denmark (DTU), 2800, Kgs. Lyngby, Denmark (e-mail: shuang@elektro.dtu.dk; qw@elektro.dtu.dk).

M. Shahidehpour is with Robert W. Galvin Center for Electricity Innovation, Illinois Institute of Technology, Chicago, IL 60616 USA, and also with King Abdulaziz University, Jeddah 21589, Saudi Arabia (e-mail: ms@iit.edu).

Corresponding author: Q. Wu (e-mail: qw@elektro.dtu.dk).

$\begin{array}{ll}V_{0} & \begin{array}{l}\text { voltage at node } 0 \\ Y_{L L}\end{array} \\ \begin{array}{ll}\text { the matrix obtained by removing the first row and } \\ \text { column of the nodal admittance matrix } \\ \text { the inverse matrix of } Y_{L L}\end{array} \\ c_{t} & \begin{array}{l}\text { forecast baseline energy price } \\ d_{i, t}\end{array} \\ e_{i}^{\min } & \text { discharging power of EVs due to driving } \\ e_{i}^{\max } & \text { upper limit of the SOC level } \\ e_{i, 0} & \text { initial SOC level } \\ p_{i, t}^{\min }, p_{i, t}^{\max } & \text { lower/upper charging power limit of EVs } \\ \hat{p}_{i}^{\min }, \hat{p}_{i}^{\max } & \begin{array}{l}\text { lower/upper power limit of HPs } \\ p_{t}^{c}\end{array} \\ q_{t}^{c} & \text { active conventional power at each load point } \\ u_{i, t} \in R^{m_{i}} & \text { reactive conventional power at each load point } \\ \alpha & \text { initial temperature } \\ \beta_{1} & \text { coefficient, step size } \\ \end{array}$

Variables

$p^{e v}, p^{h p}, p^{c}$ power level of EV, HP and conventional loads of a household/customer

$p_{i}, p_{i, t} \quad$ charging power of EVs of one aggregator

$\hat{p}_{i}, \hat{p}_{i, t} \quad$ power consumption of HPs of one aggregator

$r, r_{t} \quad$ energy-based regulation price, i.e., DT rates

$r^{p}, r_{t}^{p} \quad$ power-based regulation price, i.e., DPT rates

$R \quad$ matrix form of the DPT rates

$S_{t} \quad$ total apparent power at each load point

$\lambda_{t} \quad$ Lagrange multiplier (LM) of line limit constraint

$\rho_{t} \quad$ LM of load equation

$\omega_{t} \quad$ LM of voltage constraint

Other Symbols

$m_{i} \quad$ the number of customers of aggregator $i$

$n_{*} \quad$ cardinality of $N_{*}$, i.e. $n_{*}=\left|N_{*}\right|$

$\|*\|_{1} \quad$ L-1 norm of vector *

$\{*\}_{j} \quad \mathrm{j}$-th element of vector *

* element-wise conjugate of complex vector/matrix 


\section{INTRODUCTION}

$\mathrm{D}$ ISTRIBUTION network management will play a key role in future power system management, because a large number of distributed generation (DG) and flexible demands such as electric vehicles (EVs) and heat pumps (HPs) will be extensively deployed for better balance of the production and consumption in future power systems with high penetration of renewables. Consequently, congestion problems might occur in distribution networks due to simultaneous charging or discharging of flexible demands. Instead of reinforcement of distribution networks, distribution system operators (DSOs) can use smart coordination methods to avoid or mitigate the congestion. A number of such coordination methods have been proposed recently in the literature, such as direct control methods reviewed in [1] and indirect control methods, i.e., market-based methods, including the dynamic tariff (DT) [2][5], distribution locational marginal price (DLMP) [6], [7], line shadow price method [8], subsidy-based methods [9][12], multiagent system method or transactive control method [13]-[16] and probabilistic congestion management methods [17]-[20].

In all the above mentioned distributed congestion management methods, including DT, line shadow price and multiagent system method, the optimization models established at the aggregator (agent) side are all quadratic. This is because linear optimization models may have multiple solutions in responding to a given set of prices (time series), leading to unpredictable/uncontrollable demand response, which is undesirable for congestion management [4], [21]. In the DT method, quadratic terms come from the forecast price sensitivities; in the multiagent system method, the quadratic terms result from the objective to minimize the difference between the new schedule and the initial schedule. However, these requirements are not convenient in reality. For instance, it is usually very difficult to forecast price sensitivities; also, it is not logical to stick to the initial schedule since there are new set of prices and the customers should usually pursue the minimum cost, not the initial schedule. Besides, these are "assumptions" made by these methods, the aggregators (agents) may not choose to use price sensitivities or initial schedules in their objective functions at all. Therefore, it is necessary to propose a new type of congestion management price signal, by which the quadratic terms will be embedded in the optimization models at the aggregator side.

In the congestion management methods proposed in the existing literature, the congestion price, e.g., DT or the price signal in the multiagent system method, is charged to the customers without considering their power consumption levels. This does not reflect their contributions to the distribution network investment, operation and maintenance cost. As pointed out in [22], the distribution network cost mainly depends on the peak power of the customers, not their total energy consumption. The dimension of the distribution network is mainly decided by the peak power. Therefore, [22] proposed a new network tariff, namely, power band tariff, which can reflect the customers' real contributions to the network cost more reasonably. However, the simple structure of the power band tariff cannot reflect the coordination of the peak power of each individual customer. For instance, the transformer capacity is much smaller than the summation of the fuses of all customers under the transformer, because the peak power does not occur simultaneously. On the other hand, the (hourly average) peak power is evaluated monthly or quarterly, which means that the monthly (quarterly) power band tariff cannot respond to the fast dynamics of the modern power system with intermittent renewable energy and many distributed flexible demands. To overcome these drawbacks, this paper proposes a new tariff scheme, namely, dynamic power tariff (DPT), which can be employed for congestion management in distribution networks.

The benefits of employing the DPT method for congestion management are summarized as follows. Firstly, with the DPT method, there are quadratic terms (DPT rates multiply the square of the hourly average power consumption) in the total cost model of the energy consumption for a customer, which is in the objective functions of the aggregators. This is important because, in this way, the aggregators have unique (definite) responses to the price signals. Secondly, with this tariff scheme, the resulting quadratic terms in objective functions increase much faster than a linear function as with other tariff schemes. Therefore, those consuming higher power will pay much higher tariff than those consuming lower power, which reflects the real distribution of the network cost from a DSO point of view. Thirdly, the DPT method is calculated in a distributed manner, which means less forecast parameters required and higher certainty and commitment level from the aggregators (see explanation in section III). At last, DPT is time-varying (in this paper, it is assumed to be hourly based DPT, but it could have other time periods, such as 15 minutes); therefore, it can respond to fast dynamics of power systems with renewable energy and can be employed to solve congestion in distribution networks due to the large-scale deployment of EVs or HPs.

The main contributions of this paper are summarized as follows:1) Propose a power-based pricing mechanism, i.e., the DPT (Section III), which is quite different from the energybased pricing mechanisms in the existing literature, including DT [2]-[5]; 2) Formulate an iterative congestion management method based on the DPT (Section IV); 3) Perform case studies to validate the efficacy of the DPT method for congestion management.

The rest of the paper is organized as follows. The DPT concept and implementation method are described in Section III. The mathematical formulation of the DPT method and the control diagram are presented in Section IV. In Section V, case studies are presented and discussed. The paper ends with the conclusions.

\section{Dynamic Power TARIFF Method For CONGeStion MANAGEMENT}

In this section, the concept of DPT is presented. Afterwards, the implementation of the DPT concept for congestion 
management in distribution networks is discussed.

\section{A. Power Tariff Concept}

Power tariff is different from the normal energy-based network tariff. Assume that a typical household has EVs, a HP and some other conventional loads, and the hourly average power levels of them are $p^{e v}, p^{h p}$, and $p^{c}$, respectively (assume a smart meter is installed). For a customer with normal network tariff, the total tariff paid to the DSO will be $r\left(p^{e v}+p^{h p}+p^{c}\right) \Delta t$. However, for one under the power tariff scheme, the total network tariff will be $r^{p}\left(p^{e v}+p^{h p}+p^{c}\right)^{2} \Delta t$. Here, $\Delta t$ is usually the same as one planning period, which is normally a unit of time, e.g., 1 hour; therefore, $\Delta t$ can be ignored for brevity. A comparison between these two tariffs regarding cost calculation is shown in Table I. The DT and all other types of congestion prices from the existing congestion management methods, such as line shadow price method [8], the multiagent method [13]-[16], has a tariff per energy unit as $r$, while the power tariff has a tariff per energy unit as $r^{p}\left(p^{e v}+p^{h p}+p^{c}\right)$. It can be seen that the total network cost under the power tariff scheme will have quadratic terms, which is very important in the distributed congestion management method as it can avoid multiple solution problems of linear optimization models [4], [21]. In addition, the network cost increases faster under the power tariff scheme than under the normal tariff scheme, which is illustrated in Fig. 1. Customers with power less than the break-even power $p$ pay less under the power tariff scheme than under the normal tariff scheme; while customers with power higher than $p$ pay more under the power tariff scheme than under the normal tariff scheme.

TABLE I

COMPARISON OF DPT AND DT REGARDING COST CALCULATION

\begin{tabular}{l|c|c}
\hline & DPT & $\begin{array}{c}\text { DT and other types of } \\
\text { congestion price }\end{array}$ \\
\hline $\begin{array}{l}\text { Power tariff } \\
\text { (DKK/kW/ } \\
\mathbf{k W h}\end{array}$ & $r^{p}$ & - \\
\hline $\begin{array}{l}\text { Tariff per } \\
\text { unit energy } \\
\text { (DKK/kWh) }\end{array}$ & $r^{p}\left(p^{e v}+p^{h p}+p^{c}\right)$ & $r$ \\
\hline $\begin{array}{l}\text { Energy } \\
\text { consumed } \\
\text { (kWh) }\end{array}$ & $\left(p^{e v}+p^{h p}+p^{c}\right) \Delta t$ & $\left(p^{e v}+p^{h p}+p^{c}\right) \Delta t$ \\
\hline $\begin{array}{l}\text { Total tariff } \\
\text { cost (DKK) }\end{array}$ & $r^{p}\left(p^{e v}+p^{h p}+p^{c}\right)^{2} \Delta t$ & $r\left(p^{e v}+p^{h p}+p^{c}\right) \Delta t$ \\
\hline
\end{tabular}

It is worth mentioning that the power tariff scheme can be combined with other tariff schemes. For instance, a customer can choose the normal tariff scheme (e.g., the flat tariff rate) for its nonflexible demands, because it doesn't want to change the consumption pattern for the purpose of comfort/convenience; meanwhile, it can choose the power tariff scheme for its flexible demands to reduce cost and support the power grid. It can also be the case that, some customers choose the power tariff, while others choose the normal tariff.
Due to the limited space, this paper will not go into details about all the combinations, which is straightforward after comprehending one of them. In the following, the focus is the case that one customer chooses flat tariff for its nonflexible demands (which will be dropped in objective functions, since they are constant) and power tariff for flexible demands.

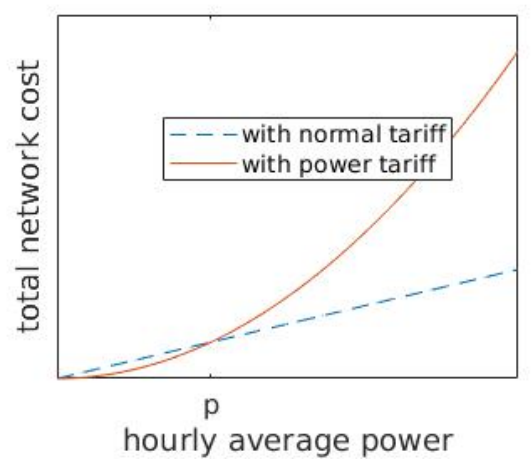

Fig. 1. Growing speed of total cost with power tariff and normal tariff

\section{B. Congestion Management through Power Tariff}

\section{1) Implementation Scenario One: $F P T+D T$}

In this scenario, power tariff has a fixed rate, i.e., fixed power tariff (FPT). Employing FPT will lead to quadratic terms in aggregator side optimization models, which can replace the price sensitivity terms required by the DT method [4]. Therefore, FPT can be combined with the DT method to solve congestion. Due to the space limit, this paper will not discuss this implementation scenario in detail. This implementation scenario is similar to the original DT method [4], except that the price sensitivity terms are replaced by the FPT terms.

2) Implementation Scenario Two: DPT

In this scenario, power tariff has a dynamic rate (varying with location and time period), i.e., DPT. This scenario is the focus of the rest of the paper. DPT can replace DT completely in congestion management of distribution networks, as it creates the same incentives as the DT method does for the customers/aggregators to reschedule their energy planning and avoid/alleviate potential congestion during planning stage.

DPT does not contain energy prices. Therefore, the aggregators, who represent the owners of the flexible demands, need to buy electricity from an electricity (energy) market, such as the day-ahead spot market in Nordic (Nord pool) or the day-ahead market of the California ISO in USA. An important task in the implementation of the DPT method for congestion management is to determine a proper DPT rate for each node (load bus) and each time period according to the network conditions and forecasted nonflexible load level, so that the energy schedules of the aggregators respect the network limits. As the DPT method works together with the dayahead spot market, the time periods for the DPT rates are typically 24 hourly based periods of the next day, i.e., in line with the day-ahead market. With the DT method, the DT rates are determined by the DSO through a centralized optimization. Unlike the DT method, DPT rates are determined by iterative 
interactions between the DSO and aggregators. The detailed procedure of implementing the DPT method for congestion management is illustrated in Fig. 2. Step 1, the DSO initiates the iterative process by sending out tentative DPTs $(=0)$. Step 2 , the aggregators will separately make their own optimal purchasing plans by minimizing the energy cost and the network tariff cost. Step 3, the aggregators will send back the tentative demand responses (DRs) to the DSO. Step 4, with the new information, the DSO will be able to identify the congestion points (if any) and modify the DPT rates according to the identified congestion. Step 5, the DSO sends out the new DPTs to the aggregator and the iterative process continues (go back to Step 2) till the network constraints are satisfied and the final DPT rates are determined. As in many other congestion management methods, Step 4 is to identify the congestion risk and allocate the risk to the responsible party through the DPT calculation (the DPT is also a locational price). Step 2 is to optimize the energy consumption plan by each aggregator taking into account the congestion risk reflected in the DPT. Steps 2 5 form an iterative process to finally reach an optimal planning with respect to the network constraints.

In the DPT method, the overall optimization problem is not known to the DSO or the aggregators. Each aggregator optimizes its own planning problem, which is smaller and easier to solve. In this sense, the DPT method is a distributed optimization method. In the DPT method, it is required that the aggregators keep the energy consumption level within the capacity that is revealed in the last iteration DR. In this sense, congestion management by the DPT method is more certain (the DSO is more confident about the congestion management result) and transparent (easy to be understood/accepted by the participants) than the normal DT method.

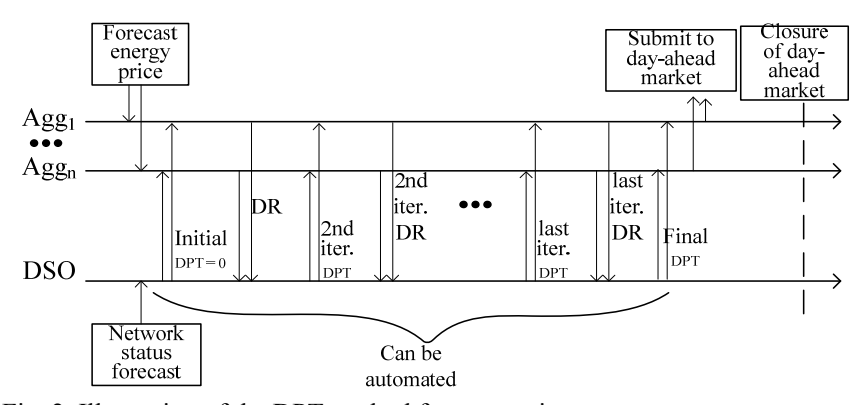

Fig. 2. Illustration of the DPT method for congestion management

\section{Calculation of Dynamic Power Tariff Rates}

\section{A. Mathematical Formulation of DPT Method}

1) Formulation at the aggregator side:

In the DPT method, the optimizations at the aggregator side are important and part of the determination of the DPT rates. It is assumed that the aggregators are purely economic units and have no information of the network conditions. Therefore, they cannot directly consider any of the network constraints. They make energy schedules based on the requirements of the flexible demands and prices, including the forecasted energy prices, and the DPTs received from the DSO. Other fixed cost (such as taxes) will not be considered since it does not change the optimal energy schedule decisions (or can be merged into the forecast energy prices). In order to facilitate the study, residential EVs and HPs are chosen to be the flexible demands. Many other types of flexible demands in reality can be well represented by the combination of EVs and HPs.

An aggregator can use a quadratic function to represent the total cost including energy consumption and network tariff as shown in (1). The quadratic term in (1) is due to the DPT received from the DSO ( $R_{t}$ is the DPT; therefore, it is a fixed parameter for the optimizations at the aggregator side). Unlike the DT method, which requires price sensitivity coefficients by forecasting in order to have a quadratic programming formulation [4], the DPT method can have a quadratic programming formulation by the power tariff concept. The optimal planning problem for aggregator $i$ is formulated as (1)-(5).

$$
\begin{array}{r}
\min _{p_{i, t}, \hat{p}_{i, t}} \sum_{t \in N_{T}}\left(p_{i, t}+\hat{p}_{i, t}\right)^{T} E_{i}^{T} R_{t} E_{i}\left(p_{i, t}+\hat{p}_{i, t}\right)+ \\
\sum_{t \in N_{T}}\left(c_{t} \mathbf{1}\right)^{T}\left(p_{i, t}+\hat{p}_{i, t}\right)
\end{array}
$$

subject to,

$$
\begin{gathered}
e_{i}^{\min } \leq \sum_{t_{-} \leq t}\left(p_{i, t_{-}}-d_{i, t_{-}}\right)+e_{i 0} \leq e_{i}^{\max }, \forall t \in N_{t},\left(\mu_{i, t}^{-}, \mu_{i, t}^{+}\right), \\
p_{i, t}^{\min } \leq p_{i, t} \leq p_{i, t}^{\max } \quad \forall t \in N_{T}, \\
K_{i, t}^{a, \min } \leq \sum_{t_{-} \leq t} A_{i, t, t_{-}} \hat{p}_{i, t_{-}}+u_{i, t} \leq K_{i, t}^{a, \max }, \forall t \in N_{T}, \\
\hat{p}_{i, t}^{\min } \leq \hat{p}_{i, t} \leq \hat{p}_{i, t}^{\max } \quad t \in N_{T},\left(\hat{\zeta}_{i, t}^{-}, \hat{\zeta}_{i, t}^{+}\right) .
\end{gathered}
$$

Constraints (2) - (3) are from the limits of EVs. Constraint (4) represents the thermal limits of households and (5) gives the input power limits of HPs. Constraint (4) is derived from the thermal process analysis of the household and the HP as shown in [4].

After solving the optimal problem, the aggregator $i$ will have a tentative optimal plan $\left(p_{i, t}^{*}, \hat{p}_{i, t}^{*}\right)$. Then $(6)$ can be used to formulate the aggregated DR for aggregator $i$, which will be sent to the DSO.

$$
p_{i, t}^{a}=E_{i}\left(p_{i, t}+\hat{p}_{i, t}\right), \forall t \in N_{t}
$$

2) Formulation at the DSO side:

At the DSO side, there is no optimization model involved, but the network limits are checked. After receiving the DR results, $p_{i, t}^{a}$, from all of the aggregators, the DSO will firstly determine the total apparent power load, $s_{t}$, for each bus using (9) and (10). Then, it will use a dc load flow method to determine the power flow of each feeder and the voltage level of each bus by (7) and (8), respectively. The method to estimate an approximate voltage level for each bus (the left side of (8)) is proposed by [23]. The inequalities of (7) and (8) are to compare the power flow and voltage level with the network limits, respectively.

$$
D \operatorname{Re}\left(s_{t}\right) \leq F_{t}, \forall t \in N_{T},\left(\lambda_{t}\right)
$$




$$
\begin{gathered}
1-\frac{1}{V_{0}^{2}} \operatorname{Re}\left(Z s_{t}\right) \geq \frac{\underline{V}}{V_{0}}, \forall t \in N_{T},\left(\omega_{t}\right) \\
\operatorname{Re}\left(s_{t}\right)=p_{t}^{c}+\sum_{i \in N_{B}} p_{i, t}^{a}, \forall t \in N_{T},\left(\rho_{t}\right) \\
\operatorname{Im}\left(s_{t}\right)=q_{t}^{c}, \forall t \in N_{T}
\end{gathered}
$$

The Matrix $Z$ is the inverse matrix of the partial nodal admittance matrix $Y_{L L}$, which is a submatrix of the full admittance matrix ( $Y_{00}$ represents the slack bus),

$$
Y=\left[\begin{array}{ll}
Y_{00} & Y_{0 L} \\
Y_{L 0} & Y_{L L}
\end{array}\right] .
$$

Vectors $\lambda_{t}$ and $\omega_{t}$ in (7) and (8) can be deemed as 'marginal' prices of the network cost with respect to power flow limits and voltage limits, respectively. The marginal prices are positive if the corresponding network constraint has an effect on the DR of the aggregators, i.e., when the constraint is binding; otherwise, it will be zero. Even though the DSO does not need to model an optimization problem, it should check the network limits by establishing and evaluating the network constraints (7) and (8).

Vectors $\lambda_{t}$ and $\omega_{t}$ play an important role in determining proper DPT rates, i.e., $R_{t}$. They are determined iteratively. Initially, they are zero, i.e., $\lambda_{t}^{(1)}=0, \omega_{t}^{(1)}=0$ and $R_{t}^{(1)}=0$. Then after receiving DR results $p_{i, t}^{a(k)}$ from the aggregators ( $k$ refers to the $k$-th iteration, and $p_{i, t}^{a(k)}$ is the optimal solution based on the $k$-th DPT, i.e., $\left.R_{t}^{(k)}\right), s_{t}^{(k)}$ can be determined. Then, $\lambda_{t}, \omega_{t}$ and $R_{t}$ can be updated by,

$$
\begin{gathered}
\lambda_{t}^{(k+1)}=\lambda_{t}^{(k)}+\alpha\left(D \operatorname{Re}\left(s_{t}^{(k)}\right)-F_{t}\right), \forall t \in N_{T}, \\
\omega_{t}^{(k+1)}=\omega_{t}^{(k)}+\alpha\left(-1+\frac{1}{V_{0}^{2}} \operatorname{Re}\left(Z \overline{s_{t}^{(k)}}\right)+\frac{\underline{V}}{V_{0}}\right), \forall t \in N_{T} \\
R_{t}^{(k+1)}=D^{T} \lambda_{t}^{(k+1)}+\frac{\operatorname{Re}\left(Z^{T}\right)}{V_{0}^{2}} \omega_{t}^{(k+1)}, \forall t \in N_{T} .
\end{gathered}
$$

In (11) and (12), $\alpha$ represents a proper step size, and the term after $\alpha$ is the residual of constraints (7) and (8), respectively. The justification of (11)-(13) will be discussed in Section IV.B by a metaphor based on the control theory. There is an implicit requirement for the marginal prices $\lambda_{t}$ and $\omega_{t}$, i.e., they must be nonnegative; therefore, they are modified by,

$$
\begin{aligned}
& \lambda_{t}=\left(\lambda_{t}\right)^{+}, \\
& \omega_{t}=\left(\omega_{t}\right)^{+},
\end{aligned}
$$

i.e., if they are negative, they will be replaced with zero.

When the iteration converges, the residuals in (11) and (12) will be nonpositive, which means constraints (7) and (8) are satisfied. There are $\left|\lambda_{t}^{(k+1)}-\lambda_{t}^{(k)}\right| \leq \tau,\left|\omega_{t}^{(k+1)}-\omega_{t}^{(k)}\right| \leq \tau$ and $\left|R_{t}^{(k+1)}-R_{t}^{(k)}\right| \leq \tau$, where $\lambda_{t}$ and $\omega_{t}$ are modified values using (14) and (15), and $\tau$ is a small tolerance. The final DPT rates are the same as the last iteration DPT rates (Fig. 2).

Although the term 'marginal price', e.g., $\lambda_{t}$ and $\omega_{t}$, is used in this subsection to explain the calculation of the DPT, it is not the same as the one (dual variable, or Lagrange multiplier) in the duality theory of optimization [24], since there is no optimization involved at the DSO side. Besides, even though one can establish an overall optimization at the DSO side like the one for the DT method [4], its Lagrange multipliers can only be employed to calculate DT rates, but not the DPT rates. In next subsection (IV.B), the marginal prices and the DPT will be explained with the knowledge of the control theory.

\section{B. Control Diagram of the Iteration Process}

From the DSO point of view, the price-based congestion management process is an indirect control process. The aggregators respond to the price signals from the DSO (e.g., DT or DPT), and the DSO can adjust the price signals such that the responses from the aggregators can respect the network limits.

The control framework of the whole congestion management process is shown in Fig. 3. The target system comprises of the distribution network and two actors, i.e., the DSO and aggregators. The aggregators receive the DPTs and make their own optimal planning (DR). Then, the planning results are sent to the DSO. After gathering the planning results from the aggregators, the DSO performs load flow analysis and determines the network status, including line flows and voltage profiles. Then, the power flows are sent to the power flow controller (upper part of the diagram), and the voltage profiles are sent to the voltage controller (lower part of the diagram).

The received signals (flows and voltages) are compared with the references respectively, and then the error signals are multiplied by the step size $\alpha$. Then the signals enter two integrators, which are corresponding to (11) and (12), respectively. Afterwards, two limiters force the signals to be nonnegative, which are corresponding to (14) and (15), respectively.

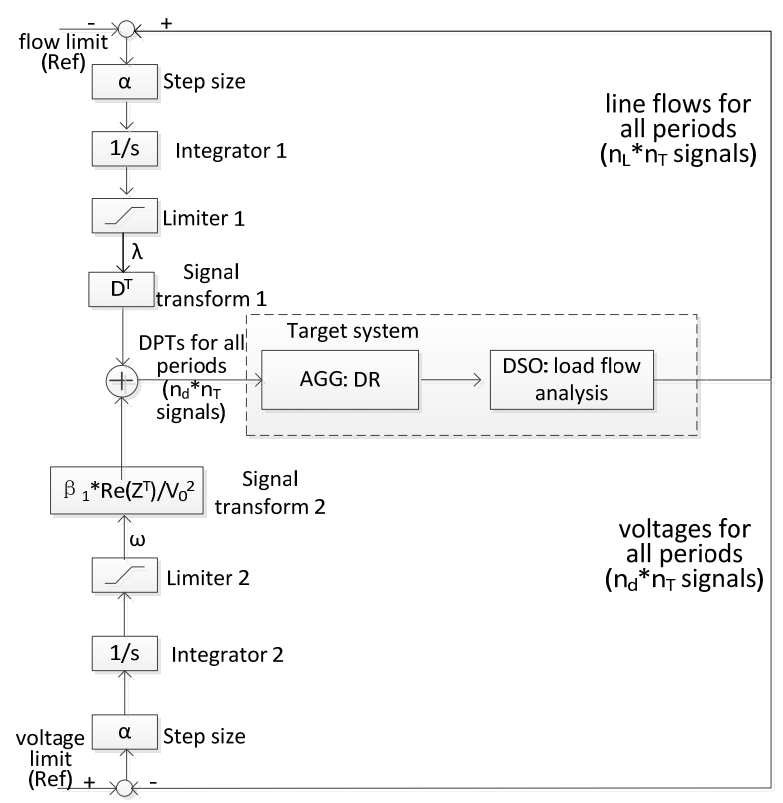

Fig. 3. Illustration of the control framework 
Then the signals (marginal prices for line flow and voltage respectively) are transformed to DPTs by the matrix $D^{T}$ and matrix $\operatorname{Re}\left(Z^{T}\right) / V_{0}^{2}$, respectively. However, signals from the voltage controller are much smaller than those from the power flow controller due to, e.g., different units. It is necessary to magnify the signals from the voltage controller by a factor $\left(\beta_{1}\right.$, see 'signal transform 2 ' in Fig. 3 ) to be comparable to those from the power flow controller. Otherwise, the voltage controller will be too slow and the overall control process will slow down. Due to the two limiters, the references are not followed unless the corresponding signals ( $\lambda$ and $\omega$ in Fig. 3) are positive. This means that when there is congestion, the marginal price and DPT will be positive and the corresponding power flow limit or voltage limit will be binding. This actually is consistent with the conclusion in the optimization theory [24].

\section{CAse Studies}

\section{A. Case study parameters}

The single line diagram of the Bus 4 distribution network of the Roy Billinton Test System (RBTS) [25] (shown in Fig. 4 ) is chosen for the case study. Line segments of the feeder one are labeled as shown in Fig. 4, among which L2, L4, L6, L8, L9, L11, and L12 refer to the transformers connecting the corresponding load points (LP1 to LP7). There are 38 load points (LP1 LP38) in total. The data of these load points are listed in Table II. Assume that the DSO has improved the power factor of the conventional consumption by reactive power compensation, and the remaining reactive power consumption is $10 \%$ of the conventional active power consumption.

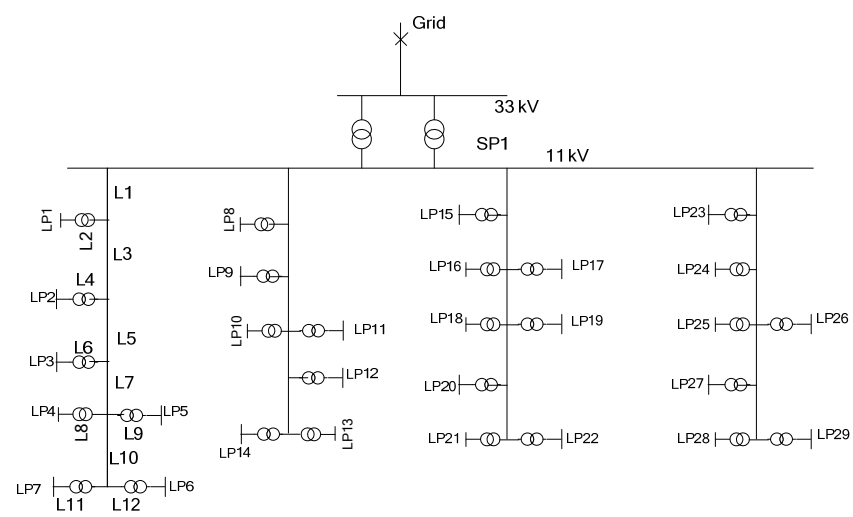

Fig. 4. Single line diagram of the distribution network

TABLE II

LOAD POINT DATA

\begin{tabular}{lllll}
\hline \multicolumn{1}{c}{ load points } & $\begin{array}{c}\text { customer } \\
\text { type }\end{array}$ & $\begin{array}{c}\text { peak conv. } \\
\text { act. power } \\
\text { per point } \\
\mathbf{( k W )}\end{array}$ & $\begin{array}{c}\text { peak } \\
\text { conv. } \\
\text { react. } \\
\text { (kVar) }\end{array}$ & $\begin{array}{c}\text { number } \\
\text { of cus- } \\
\text { tomers } \\
\text { per point }\end{array}$ \\
\hline LP1-4,LP8-11, & & & & \\
LP15-19,LP23-26 & residential & 886.9 & 88.69 & 200 \\
\hline LP5,LP12,LP20,LP27 & residential & 813.7 & 81.37 & 200 \\
\hline LP6,LP7,LP13,LP14, & & & & \\
LP21,LP22,LP28,LP29 & commercial & 671.4 & 67.14 & 10 \\
\hline
\end{tabular}

The key parameters of the simulation are listed in Table III. The lower voltage limit is set to be $0.948 \mathrm{p}$.u. in order to have a small margin (0.006 0.008 p.u.) compared to the assumed physical limit 0.94 p.u. The EV availability shown in Fig. 5 is from the driving pattern study in [26]. The household area is a random number between 100 and $200\left(\mathrm{~m}^{2}\right)$.

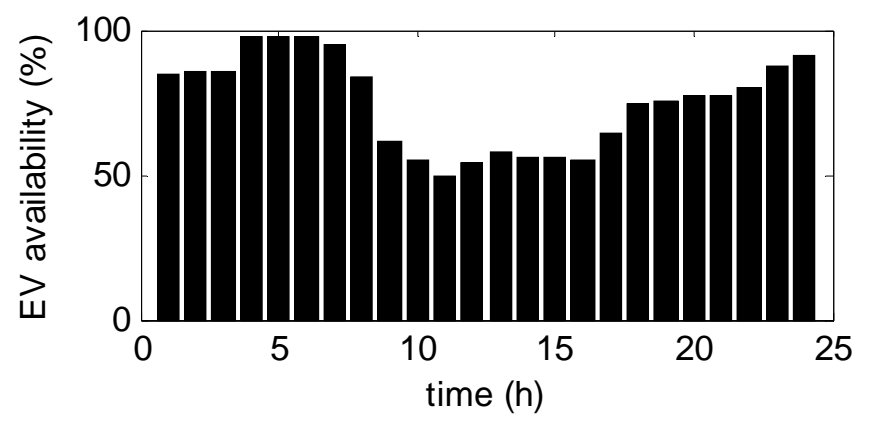

Fig. 5. EV availability

TABLE III

Key Parameters of the Simulation Model ([26], [27])

\begin{tabular}{ll}
\multicolumn{1}{c}{ parameter } & \multicolumn{1}{c}{ value } \\
\hline EV battery size & $25 \mathrm{kWh}$ \\
\hline Peak charging power & $11 \mathrm{~kW}(3$ phase $)$ \\
\hline Energy consumption per $\mathrm{km}$ & $150 \mathrm{Wh} / \mathrm{km}$ \\
\hline Minimum SOC & $20 \%$ \\
\hline Maximum SOC & $85 \%$ \\
\hline Average driving distance & $40 \mathrm{~km}$ \\
\hline COP of HP & 2.3 \\
\hline Min Temp. of the House & $20{ }^{\circ} \mathrm{C}$ \\
\hline Max Temp. of the House & $24{ }^{\circ} \mathrm{C}$ \\
\hline Voltage rating $=V_{0}$ & $11 \mathrm{kV}$ \\
\hline Lower voltage limit & $0.948 \mathrm{p.u}$. \\
\hline L2 limit $(\mathrm{kW})$ & 1100 \\
\hline L3 limit $(\mathrm{kW})$ & 7000 \\
\hline L4 limit $(\mathrm{kW})$ & 2700 \\
\hline$\alpha$ & 0.6 \\
\hline$\beta_{1}$ & 10 \\
\hline $\mathrm{x} / \mathrm{r}$ ratio of line & $0.4 \sim 0.6$ \\
\hline $\mathrm{x} / \mathrm{r}$ ratio of transformers & $\approx 6$ \\
\hline
\end{tabular}

\section{B. Case study results}

The simulation was carried out using the GAMS/CPLEX optimization software [28] for the DR (optimization) at the aggregator side, and a Matlab script for the iteration control at the DSO side, including the DPT calculation and convergence check.

1) Congestion Management Results:

Firstly, the DR at the aggregator side was performed with initial zero DPT (the forecast system prices (base price) are the same as in case studies for the DT method, which can be seen in Fig. 12). The line loading profiles were determined by the DSO after receiving DRs from the aggregators. As shown in Fig. 6, there is congestion at hours 17, 18, 19 and 24. Then the iteration starts. When the iteration converges, the final DPT can be determined and is shown in Fig. 7 (Feeder 1) and Fig. 8 (Feeder 3). The line/transformer loadings of L2, L3 and L4 and the voltage profile of a critical bus (LP4) after using 
the DPT method are shown in Fig. 9 and Fig. 10, respectively. It is shown that both line/transformer loadings and voltage profile are within the allowed limits.
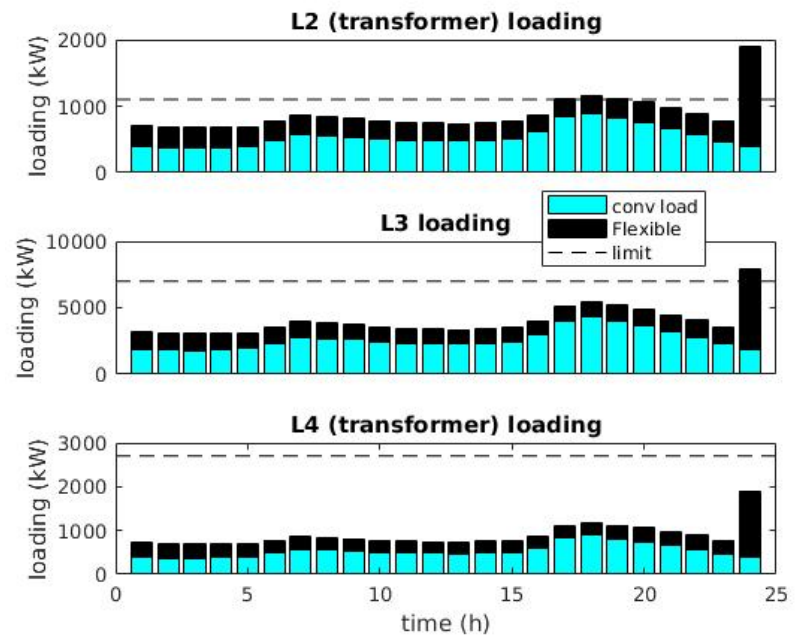

Fig. 6. Line loading results with the initial DPT $=0$

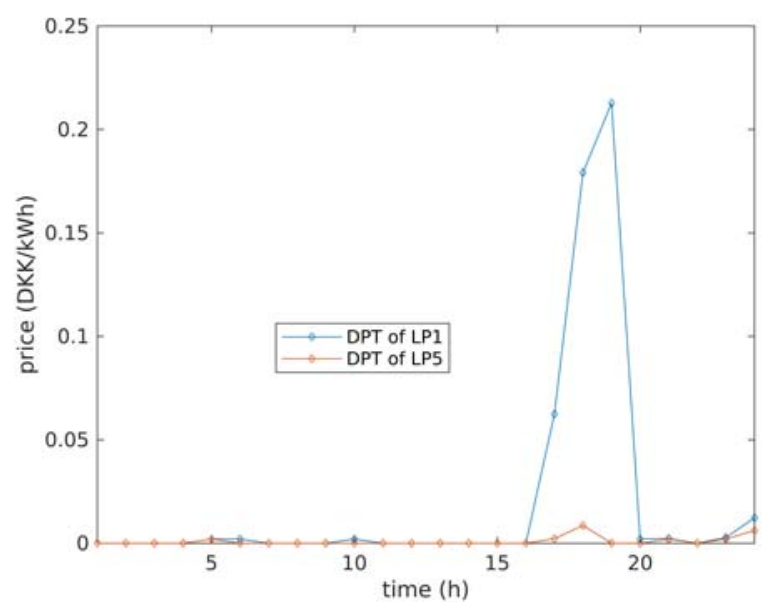

Fig. 7. DPT for different load points of Feeder 1 (DPTs for LP2-4 are the same as LP5)

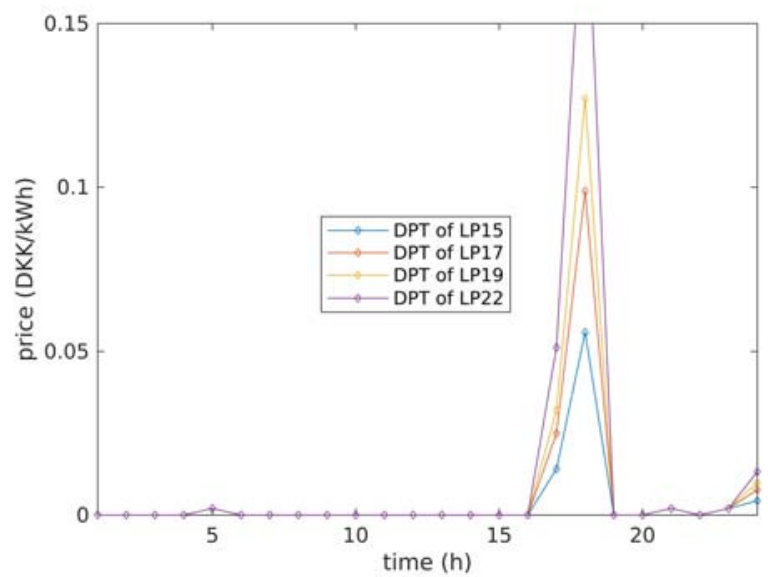

Fig. 8. DPT for different load points of Feeder 3

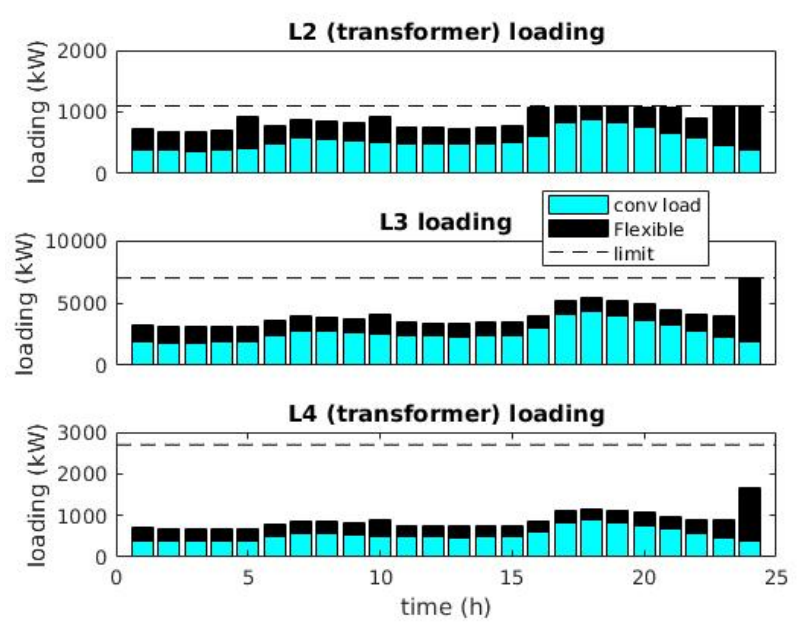

Fig. 9. Line loading results after using the DPT method

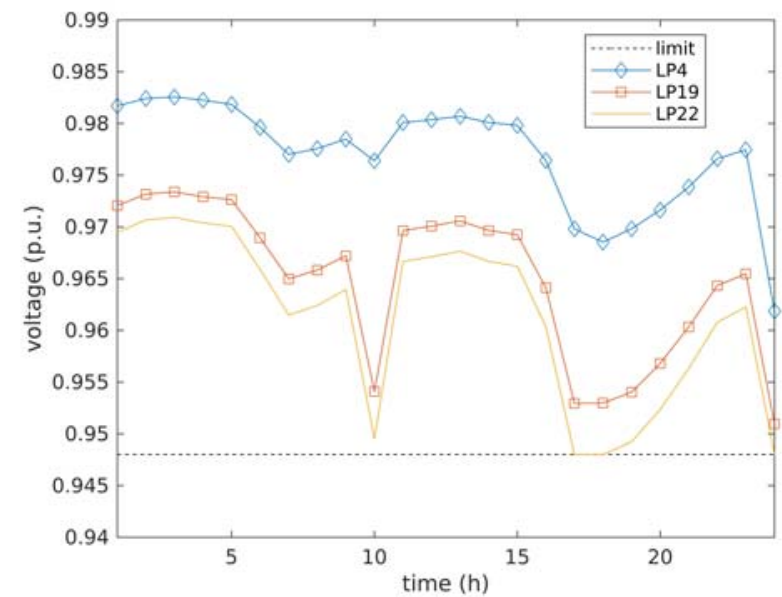

Fig. 10. Voltage profile of LP4, LP19 and LP22

2) Comparison among Households:

In order to support the second statement made in Section II regarding the benefits of using DPT method, i.e., the higher power household pays much more tariff than the lower power household, the detailed simulation results of the first 50 households on LP19 are studied. Among the 50 households, the house sizes vary between 100 200 $\mathrm{m}^{2}$ (affect the HP power) and the EV energy demand varies between $1 \sim 20 \mathrm{kWh}$ per day. The results are shown in Fig. 11, where one can see that the total tariff increases much faster than linear growth as normally seen in other congestion pricing schemes, including the DT method.

3) Comparison with DT Method:

A comparison between the DPT method and the DT method for congestion management was conducted. The DT method was employed for congestion management of the same network and conditions (EVs and HPs). As mentioned in Section II, the price sensitivity plays an important role in the DT method. Therefore, the DT method was employed under different assumptions about the price sensitivity, i.e., sensitivity $=0.0001,0.001,0.01,0.1$, respectively. The resulting DTs (for LP22) can be seen in Fig. 12, which clearly shows the 
impact of the price sensitivity coefficients on the DTs, especially when the sensitivity $\geq 0.01$ (the DTs are quite similar when sensitivity $\leq 0.01$; therefore, not shown in the figure). They are different for the congestion hours, i.e., hour 18, 19 and 24. This implies uncertainties about the congestion management results by the DT method, as different aggregators may choose different price sensitivities as they want (including zero, which may lead to failure of the DT method).

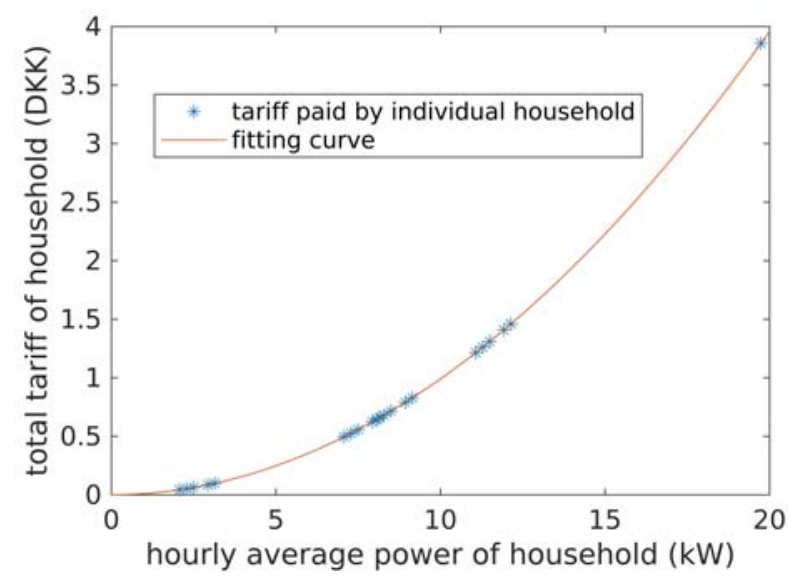

Fig. 11. Total paid tariff of each individual household on LP19 at hour 24

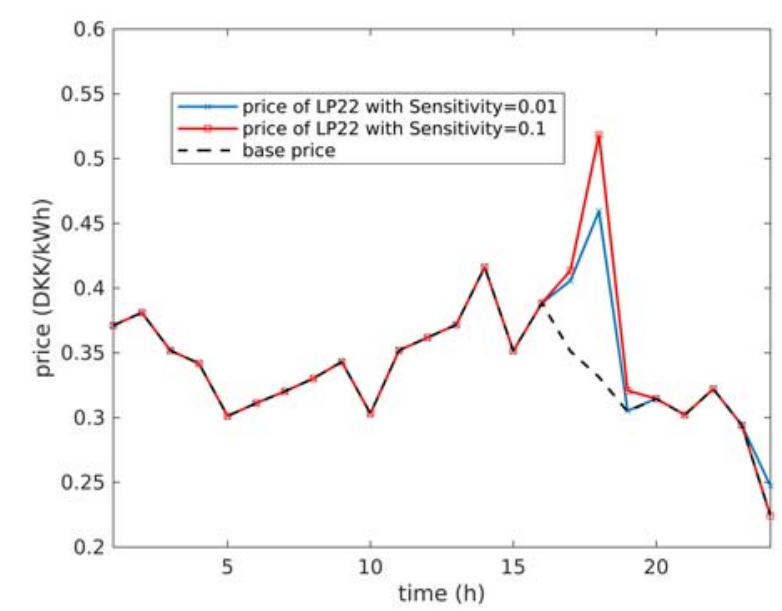

Fig. 12. DT method: Forecast system prices (base price) and final prices (including DT with different sensitivity coefficients) with respect to different sensitivities.

\section{4) Convergence Observation:}

The key variables, including the line flow and voltage, the corresponding marginal prices $\lambda_{t}$ and $\omega_{t}$, and the DPT rates, are observed in the iteration process. The results are shown in Fig. 13. It can be seen that $\lambda_{t}$ is settled down after 50 iterations and $\omega_{t}$ is settled down after 400 iterations, because the voltage controller is a bit slower than the power flow controller for the purpose of avoiding strong interference between the two controllers. Also, the consecutive congestion at hour 17, 18 and 19 makes is very challenging for the control to settle down. If there is only a single congestion hour, the control can settle down after about 10 iterations. In practice, the iteration process should be automated. The overall time for the congestion management using the DPT method is depending on how many iterations are needed for convergence and the time needed for one communication between the DSO and aggregators. The optimization at each aggregator is very fast (less than 0.5 second in this simulation) because the problem is fully decomposable.
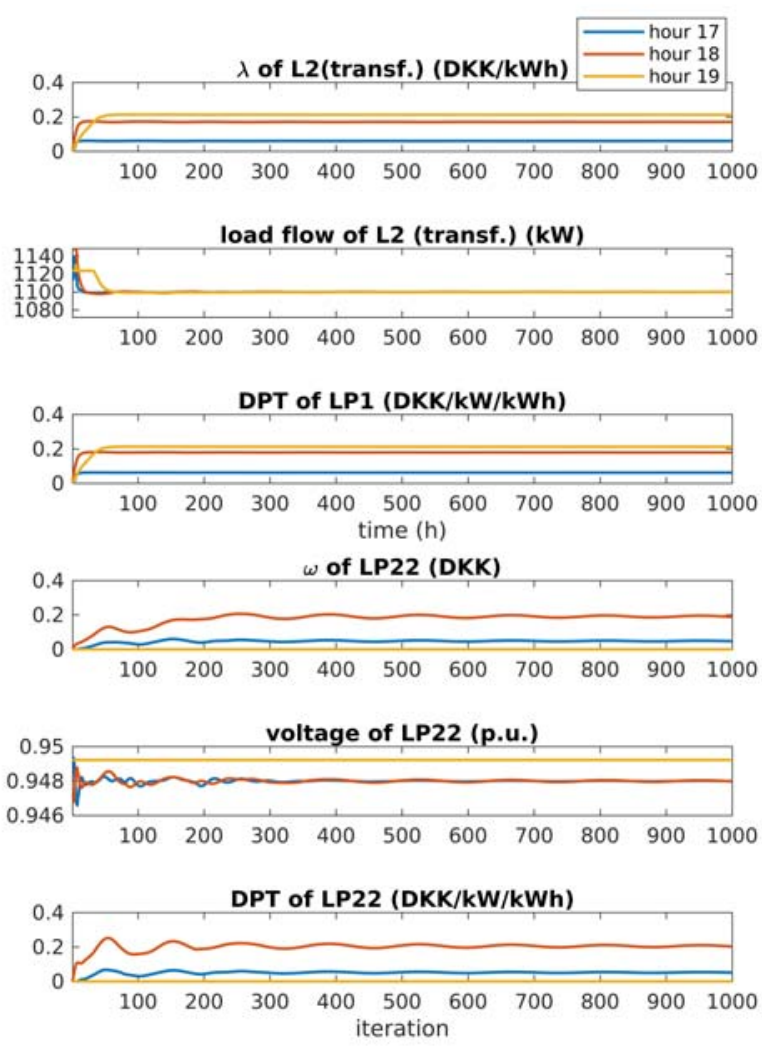

Fig. 13 Iteration observation of some key variables

\section{5) Other Discussions:}

At the DSO side, the DSO only needs to forecast the conventional active and reactive power consumption of each load points with the DPT method. It doesn't need to forecast the price sensitivities or the flexible demands as in the DT method. At the aggregator side, the forecast is almost the same for the DPT method and the DT method. Depending on the contracts between the aggregators and their customers, the aggregators may need to forecast the demand level and the availability of the flexible demands, or their customers report this information to them. Therefore, the DPT method needs less forecasting than the DT method in general.

When the distribution system becomes larger and there are more households (more EVs and HPs), the congestion management with the DPT method does not necessarily become more complicated or need more computation time for the following two reasons. Firstly, the computation at the DSO side is only algebra calculation (no optimization involved), which takes almost no time. The computation at the aggregator side, 
i.e., the optimization, is totally decomposable because there is no coupling constraint, such as network constraints. The optimization can be done in parallel for each household and then the results can be summed up. Secondly, the iteration number is very much related to how many congestion points and hours in the distribution networks, not the size of the network or the number of variables. Therefore, the DPT method can be easily employed for a scaled-up system.

\section{CONCLUSIONS}

This paper proposes the DPT method for congestion management in distribution networks with high penetration of EVs and HPs. The DPT method employs the power tariff concept, leading to a quadratic programing problem in the optimizations at the aggregator side without using price sensitivity coefficients as in the DT method. In addition, the DPT method is implemented through an iteration process, which can have the aggregators participate in the congestion management explicitly, implying more certainty and commitment level from the aggregators. The case studies have validated the efficacy of the DPT method for congestion management, and shown its ability of saving network tariff cost for the customers with lower power consumption level.

\section{REFERENCES}

[1] S. Huang, Q. Wu, Z. Liu, and A. H. Nielsen,"Review of congestion management methods for distribution networks with high penetration of distributed energy resources," in Proc. 2014 IEEE PES Innovative Smart Grid Technologies, Europe, pp. 1-6.

[2] N. O'Connell, Q. Wu, J. Østergaard, A. H. Nielsen, S. T. Cha, and Y. Ding,"Day-ahead tariffs for the alleviation of distribution grid congestion from electric vehicles," Electr. Power Syst. Res., vol.92, pp. 106-114, 2012

[3] R. Li, Q. Wu, and S. S. Oren, "Distribution locational marginal pricing for optimal electric vehicle charging management," IEEE Trans. Power Syst., vol.29, no.1, pp. 203-211, Jan. 2014.

[4] S. Huang, Q. Wu, S. S. Oren, R. Li, and Z. Liu,"Distribution locational marginal pricing through quadratic programming for congestion management in distribution networks," IEEE Trans. Power Syst., vol.30, no.4, pp. 2170-2178, Jul. 2015.

[5] S. Huang, Q. Wu, L. Cheng, and Z. Liu, “Optimal reconfiguration-based dynamic tariff for congestion management and line loss reduction in distribution networks," IEEE Trans. Smart Grid, vol.7, no.3, pp. 12951303, May 2016.

[6] P. M. Sotkiewicz and J. M. Vignolo,"Nodal pricing for distribution networks: Efficient pricing for efficiency enhancing DG," IEEE Trans. Power Syst., vol.21, no.2, pp. 1013-1014, May 2006.

[7] G. T. Heydt, B. H. Chowdhury, M. L. Crow, D. Haughton, B. D. Kiefer, F. Meng, and B. R. Sathyanarayana,"Pricing and control in the next generation power distribution system," IEEE Trans. Smart Grid, vol.3, no.2, pp. 907-914, Jun. 2012.

[8] B. Biegel, P. Andersen, J. Stoustrup, and J. Bendtsen,"Congestion management in a smart grid via shadow prices," in Proc. 2012 8th IFAC Symposium on Power Plant and Power System Control, pp. 518-523.

[9] M. R. Sarker, M. A. Ortega-Vazquez, and D. S. Kirschen,"Optimal coordination and scheduling of demand response via monetary incentives," IEEE Trans. Smart Grid, vol.6, no.3, pp. 1341-1352, May 2015.

[10] H. Zhong, L. Xie, and Q. Xia,"Coupon incentive-based demand response: theory and case study," IEEE Trans. Power Syst., vol.28, no.2, pp. 1266-1276, May 2013.

[11] C. Zhang, Y. Ding, N. Nordentoft, P. Pinson, and J. Østergaard,"FLECH-A danish market solution for DSO congestion management through DER flexibility services," J. Mod. Power Syst. Clean Energy, vol.2, no.2, pp. 126-133, 2014.
[12] S. Huang and Q. Wu, "Dynamic subsidy method for congestion management in distribution networks," IEEE Trans. Smart Grid, pp. 1$1,2016$.

[13] B. Moradzadeh and K. Tomsovic,"Two-stage residential energy management considering network operational constraints," IEEE Trans. Smart Grid, vol.4, no.4, pp. 2339-2346, Dec. 2013.

[14] S. Weckx, R. D’Hulst, B. Claessens, and J. Driesensam,“Multiagent charging of electric vehicles respecting distribution transformer loading and voltage limits," IEEE Trans. Smart Grid, vol.5, no.6, pp. $2857-$ 2867, Nov. 2014

[15] S. Hanif, H. B. Gooi, T. Massier, T. Hamacher, and T. Reindl,"Distributed Congestion Management of Distribution Grids under Robust Flexible Buildings Operations," IEEE Trans. Power Syst., pp. 1-1, 2017.

[16] P. Bach Andersen, J. Hu, and K. Heussen, “Coordination strategies for distribution grid congestion management in a multi-actor, multiobjective setting," in Proc. 3rd IEEE PES Innovative Smart Grid Technologies Europe (ISGT Europe), pp. 1-8.

[17] M. Esmaili, N. Amjady, and H. A. Shayanfar,"Stochastic congestion management in power markets using efficient scenario approaches," Energy Convers. Manag., vol.51, no.11, pp. 2285-2293, 2010.

[18] M. Hojjat and M. H. Javidi D. B.,"Probabilistic congestion management considering power system uncertainties using chance-constrained programming," Electr. Power Components Syst., vol.41, no.10, pp. 972 989, Jul. 2013

[19] Z. Liu, Q. Wu, S. Oren, S. Huang, R. Li, and L. Cheng,"Distribution locational marginal pricing for optimal electric vehicle charging through chance constrained mixed-integer programming," IEEE Trans. Smart Grid, pp. 1-1, 2016.

[20] S. Huang, Q. Wu, L. Cheng, Z. Liu, and H. Zhao,"Uncertainty management of dynamic tariff method for congestion management in distribution networks," IEEE Trans. Power Syst., vol.31, no.6, pp. 43404347, Nov. 2016.

[21] W. Liu and F. Wen, "Discussion on 'Distribution Locational Marginal Pricing for Optimal Electric Vehicle Charging Management,"' IEEE Trans. Power Syst., vol.29, no.4, pp. 1866-1866, Jul. 2014.

[22] J. Partanen, S. Honkapuro, J. Tuunanen, and H. Niemelä. (2012). Tariff scheme options for distribution system operators. LUT. [online]. Available:

http://www.lut.fi/documents/10633/138922/Tariff+scheme+options+for +distribution+system+operators/d2c7a66f-4033-42ff-a581de4ef8586592.

[23] S. Bolognani and S. Zampieri,"On the existence and linear approximation of the power flow solution in power distribution networks," IEEE Trans. Power Syst., vol.31, no.1, pp. 163-172, Jan. 2016

[24] S. Boyd and L. Vandenberghe,Convex Optimization. Cambridge University Press, 2004.

[25] R. N. Allan, R. Billinton, I. Sjarief, L. Goel, and K. S. So,“A reliability test system for educational purposes-basic distribution system data and results," IEEE Trans. Power Syst., vol.6, no.2, pp. 813-820, May 1991.

[26] Q. Wu, A. H. Nielsen, J. Østergaard, F. Marra, and C. Træholt,"Driving pattern analysis for electric vehicle (EV) grid integration study," in Proc. 2010 IEEE PES Innovative Smart Grid Technologies Conference Europe (ISGT Europe), pp. 1-6.

[27] J. M. Nyers and A. J. Nyers, "COP of heating-cooling system with heat pump," in Proc. IEEE 3rd International Symposium on Exploitation of Renewable Energy Sources (EXPRES), pp. 17-21.

[28] R. E. Rosenthal. (2014, Aug. ). GAMS - A user's guide. GAMS Development Corporation, Washington, DC, USA. [online]. Available: http://www.gams.com/dd/docs/bigdocs/GAMSUsersGuide.pdf.

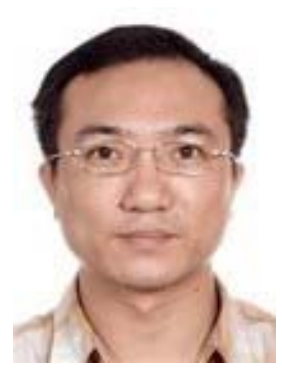

\section{BIOGRAPHIES}

Shaojun Huang (S'13) obtained the B. Eng. from Department of Electrical Engineering, Tsinghua University, Beijing, P. R. China, in 2001. He obtained the MSc from Department of Energy Technology, AAlborg University, Den-mark, in 2013.

Currently, he is pursuing his $\mathrm{PhD}$ degree in Centre for Electric Power and Energy, Department of 
Electrical Engineering, Technical University of Den-mark.

His research interests are congestion management for distribution networks with high penetration of distributed energy resources.

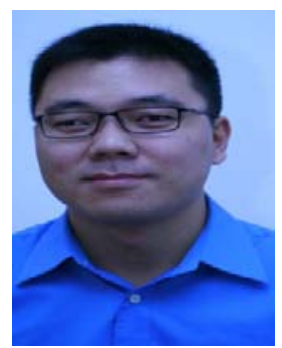

Qiuwei Wu (M'08-SM'15) obtained the B. Eng. and M. Eng. in Power System and Its Automation from Nanjing University of Science and Technology, Nanjing, China, in 2000 and 2003, respectively. He obtained the PhD degree in Power System Engineering from Nanyang Technological University, Singapore, in 2009.

He was a senior R\&D engineer with VESTAS Technology R\&D Singapore Pte Ltd from Mar. 2008 to Oct. 2009. He has been working at Department of Electrical Engineering, Technical University of Denmark (DTU) since Nov. 2009 (PostDoc Nov. 2009-Oct. 2010, Assistant Professor Nov. 2010-Aug. 2013, Associate Professor since Sept. 2013). He was a visiting scholar at Department of Industrial Engineering \& Operations Research (IEOR), University of California, Berkeley, from Feb. 2012 to May 2012 funded by Danish Agency for Science, Technology and Innovation (DASTI), Denmark. He was a visiting professor named by Y. Xue, an Academician of Chinese Academy of Engineering, at Shandong University, China, from Nov. 2015 to Oct. 2017. Currently, he is a visiting scholar at School of Engineering and Applied Science, Harvard University.

His research area is power system operation and control with high renewables, including wind power modelling and control, active distribution networks, and integrated energy systems. He is an Editor of IEEE Transactions on Smart Grid and IEEE Power Engineering Letters. He is also an Associate Editor of International Journal of Electrical Power and Energy Systems, Journal of Modern Power Systems and Clean Energy and IET Renewable Power Generation.

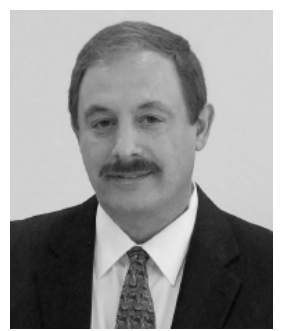

Mohammad Shahidehpour (F’01) is the Bodine Chair Professor with the Department of Electrical and Computer Engineering, the Director of the Robert W. Galvin Center for Electricity Innovation, and an Associate Director of WISER with the Illinois Institute of Technology, Chicago, IL, USA. He was a recipient of the IEEE PES Outstanding Power Engineering Educator Award, the IEEE PES Outstanding Engineer Award, and the Chicago Chapter. He is the Holder of Nourbakhshian Endowed Chair Professorship, University of Kashan, Iran, and Otto Monsted Professorship, Technical University of Denmark. He is a Research Professor with King Abdulaziz University, Saudi Arabia, the Sharif University of Technology, Iran, as well as several universities in China, including Tsinghua University, Xian Jiaotong University, Nanjing University, North China Electric Power University, and Hunan University. He is an IEEE PES Distinguished Lecturer, and served as the VP of Publications for the IEEE Power and Energy Society, the Editor-in-Chief of the IEEE TRANSACTIONS ON POWER SYSTEMS, and the Founding Editor-in-Chief of the IEEE TRANSACTIONS ON SMART GRID.

Dr. Shahidehpour is a member of the U.S. National Academy of Engineering.

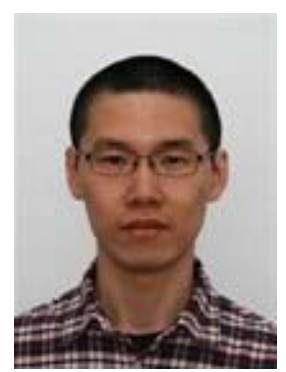

Zhaoxi Liu obtained the B. Eng. and M. Eng. from Department of Electrical Engineering, Tsinghua University, Beijing, P. R. China, in 2006 and 2008, respectively. He obtained his PhD degree at Centre for Electric Power and Energy, Department of Electrical Engineering, Technical University of Denmark in 2016.

His research interests are optimal management of electric vehicles and heat pumps in distribution networks. 